Indian J Anim Health (2021), 60(1) : 117-118

DOI: $10.36062 /$ ijah.60.1.2021.117-118

\title{
One Health needs to focus
}

\author{
Prof. Gaya Prasad ${ }^{1}$
}

${ }^{1}$ Former: Vice Chancellor, Sardar Vallabhbhai Patel University of Agriculture and Technology, Modipuram, Meerut, UP; Assistant Director General (Animal Health), ICAR, New Delhi

Every time there is an outbreak of a new disease in humans, animals are usually suspect and create some sort of discussion on how to prevent the future occurrence of new diseases. So, when, a new disease with involvement of lungs and pneumonia-like symptoms reported from Wuhan in China early in December 2019, the scientific community and people at large including politician across the globe started asking pertinent questions such as from where the causative agent, which was later identified as novel coronavirus, emerged? Was it already prevalent but identified later? The wholegenome sequence data of SARS-CoV-2 (Severe Acute Respiratory Syndrome CoronaVirus-2) indicated that it has $79.5 \%$ nucleic acid sequence homology with SARS-CoV, 97\% homology with horseshoe bat corona virus, 93.3\% nucleotide identify with RmYN02 bat coronavirus, and $91 \%$ similarity with Pangolin coronavirus. This revelation raised another question. Is bat coronavirus directly jumped into people who had come in contact with bats/ bat contaminated objects or it spilled over to some intermediary animal host which in turn infected human beings? The question of the origin of novel coronavirus which spread across all the continents and resulted in an unprecedented pandemic named Covid-19 and the novel coronavirus named SARS-CoV-2 in continuation of SARS-CoV, the coronavirus epidemic in 2003. It is our great opportunity that the epidemiological and molecular virological research has been expanded to understand the dynamics of the spread of SARS-CoV-2 in different countries. Over the past one year, quite a few reports suggested that big wild cats such as tigers and lions in zoos can be infected by infected handlers and caretakers. Similarly, pets such as cats and dogs have also been found to get the infection from infected pet owners but such reports are rare. However, there are no substantial reports to suggest that infected pets in turn can infect human beings.

The viral spillover/host switching/ jumps/cross-species transmission is a very complex process and not fully understood. However, the critical mutation in viral genes has been linked to the cross-species transmission. For example, the feline panleukopenia virus jumps to canines after certain mutations which are now known as canine parvovirus. Similarly, the simian immunodeficiency virus jumps to humans and caused the HIV pandemic. There are several such examples of host switching historically. In nature, most viruses are confined to particular hosts' species because of specific protein "lock and key" interactions. These are needed for successful replication, movement within the host, and transmission between hosts.

Production animals such as sheep, goats, cattle and buffaloes have not been reported to be susceptible to SARS-CoV-2. The devastating global impact of the Covid-19 pandemic again revived the concept of 'One Health' and we witnessed extensive discussions devoted to this theme nationally as well as internationally. Accordingly, some countries formed joint 'one health' committees but the role of veterinary experts in the committees remains marginal. World Health Organization (WHO), World Animal Health Organization (OIE) and Food and Agriculture Organization (FAO) represent a tripartite coalition to efficiently address the challenges in the human and animal health sectors under the concept of 'One Health'. 
However, wildlife and the environment are not adequately represented under 'One Health'. It is difficult to achieve the desired objectives of One Health if not adequately represented by all the stakeholders with equal say in decision making. It has been suggested by experts and concerned people across the world that the UNEP (UN Environment Program) host the 'One Health' coalition with WHO, OIE, and FAO as it does the Convention on Biological Diversity. UNEP could advocate this in a stronger voice much-needed human and animal health challenges with the sustainable development goals.

Without framing of complex 'One Health' system in an explicit manner, the ambitions implicit in the 'One Health' is unlikely to be met because of functional mismatches at the time of implementation. Public health and veterinary services should be aligned to address the issues concerning cross-sectoral health challenges. The social and ecological changes that drive new disease emergence and spread are not adequately monitored in continuous realtime surveillance. Because of the lack of extensive real-time national microbial screens, particularly in developing countries, the potential new pathogens are not detected before they spill over to humans.

The World Veterinary Association declared a theme entitled, "Veterinarian response to Covid-19 crisis". Veterinarians responded in different ways including tele-veterinary medicine formats keeping in view the coronavirus guidelines and lockdowns across the countries and helped to mitigate the suffering of animals, keeping the animal-based food supply safe as far as possible in lockdown situations, participated in multi-disciplinary research teams aimed at understanding the ecology of the pandemic and identification of drivers which might have been responsible for its emergence, offered veterinary research laboratories for processing of human diagnostic samples, complemented in the viral genomic sequencing programmes, etc. The veterinarians also participated in animal experimentation for the development of Covid-19 vaccines. The Covid19 pandemic exposed the deficiencies of the public health system in technologically advanced as well as developing countries. In India, the second wave caused enormous avoidable sufferings due to a lack of sound science-based policy interventions. If we do not learn the right kind of lessons from the pandemic, we are bound to repeat the mistakes in the future as well. The research infrastructure must be improved drastically to take it to international standards. Massive investment is required to develop modern institutions based on the 'One Health' concept and inter-instructional linkage involving expertise of medical, veterinary and environmental/ecological sciences. Multidisciplinary working groups with independent powers are needed to direct the research and development in the 'One Health'. Multidisciplinary real-time genomic surveillance of potential pathogens in multiple centres has been established in strategic geographic regions in India. It is well known that the course curriculum of veterinary and medical graduates yet does not give adequate emphasis required for developing the 'One Health' concept. Therefore, it is a national imperative that the course curriculum, if required, may be changed to give desired focus in teaching of 'One Health' at undergraduate levels. Similarly, school education should also include teaching students the concept of hygiene and infectious disease prevention. 